\title{
Drag in Paired Electron-Hole Layers
}

\author{
Giovanni Vignale \\ Department of Physics, University of Missouri, Columbia, Missouri 65211 \\ A. H. MacDonald \\ Department of Physics, Indiana University, Bloomington, Indiana 47405
}

(Received 3 October 1995)

\begin{abstract}
We investigate transresistance effects in electron-hole double-layer systems with a superfluid electronhole condensate. Our theory is based on the use of a minimum dissipation premise to fix the current carried by the condensate. We find that the drag resistance jumps discontinuously at the condensation temperature and diverges as the temperature approaches zero.
\end{abstract}

PACS numbers: 73.50.Dn

The possibility of realizing a superfluid condensate in a system composed of spatially separated layers of electrons and holes was proposed some time ago [1]. Only recently, however, has it become feasible [2-5] to produce systems where the electrons and holes are close enough together to interact strongly and at the same time sufficiently isolated to strongly inhibit optical recombination. Hopes that a superfluid electron-hole condensate might occur are supported by theoretical work in the strong magnetic field limit $[6,7]$ where some simplifications occur and the conclusion can be established with greater confidence. In this Letter we present a theory of transresistivity coefficients, which relate electric fields in one layer to currents flowing in the opposite layer, for the case of an electron-hole double-layer (EHDL) system with a superfluid electronhole condensate. We conclude that the measurement of these transport coefficients could provide an unambiguous experimental indication of the existence of the condensate. Interest in transconductance coefficients for nearby electron and hole layers [8] has increased lately [9] with the advent of accurate experimental measurements $[4,10]$. When no condensate is present, the transresistivity is typically [10] several orders of magnitude smaller than the isolated layer resistivity. In our theory, the transresistivity jumps to a value comparable to the isolated layer resistivity as soon as the condensate forms, it continues to increase with decreasing temperature $T$, and it diverges as $T \rightarrow 0$

The starting point for our theory is a minimum dissipation [11] premise which we use to fix the pair momentum of the condensed electron-hole pairs in the nonequilibrium current carrying state of the EHDL system. Using a matrix notation for the layer indices ( $e$ for electrons, $h$ for holes) we partition the total current density into a superfluid portion $\left[\mathbf{j}_{s}=\left(j_{s h}, j_{s e}\right)\right]$ carried by the condensate and a normal portion $\left[\mathbf{j}_{n}=\left(j_{n h}, j_{n e}\right)\right]$ carried by the quasiparticles:

$$
\mathbf{j}=\mathbf{j}_{s}+\mathbf{j}_{n},
$$

where the superfluid portion is proportional to the pairingmomentum $P$. ( $(\vec{P}$ will be directed along the direction of current flow.) Since it represents the flow of oppositely charged bound pairs, $\mathbf{j}_{s}=\left(P e n_{\text {pair }} / m_{\text {pair }}\right)(1,-1)$ where $n_{\text {pair }} / m_{\text {pair }}$ is defined by this equation. The physical situations of interest to us will include ones in which the sum of electron and hole currents is not zero so that $\mathbf{j}_{n}$ cannot be zero. A quasiparticle current will be generated if electric fields which drive the quasiparticles from equilibrium with the condensate are present in the electron and hole layers. In linear response

$$
\mathbf{j}_{n}=\boldsymbol{\sigma}^{\mathrm{qp}} \mathbf{E} .
$$

(Expressions for $n_{\text {pair }} / m_{\text {pair }}$ and the quasiparticle transconductivity matrix $\sigma^{\mathrm{qp}}$ based on a microscopic theory will be derived below.) A quasiparticle current flowing in the presence of electric fields will dissipate energy at a rate per unit area given by the Joule heating expression:

$$
\begin{array}{r}
W(P)=\mathbf{j}_{n} \cdot \mathbf{E}=\left[\mathbf{j}-P e n_{\mathrm{pair}} / m_{\mathrm{pair}}(1,-1)\right] \cdot \boldsymbol{\rho}^{\mathrm{qp}} \\
\cdot\left[\mathbf{j}-P e n_{\mathrm{pair}} / m_{\mathrm{pair}}(1,-1)\right],
\end{array}
$$

where $\boldsymbol{\rho}^{\mathrm{qp}}$, the quasiparticle transresistivity matrix, is the inverse of the quasiparticle transconductivity matrix. The pairing momentum of the condensate in the nonequilibrium current carrying state will be the one which allows the prescribed currents to flow through the system with minimum dissipation. Applying this condition we find a surprisingly simple result:

$$
\mathbf{j}_{s} \cdot \mathbf{E}=0 .
$$

The electric fields in electron and hole layers are identical independent of the microscopic transresistivity matrix and the prescribed currents.

Explicit expressions for $\mathbf{j}_{s}, \mathbf{j}_{n}$, and $\mathbf{E}$ can be obtained by combining Eqs. (1), (2), and (4). We find that for prescribed current $\mathbf{j}=\left(j_{h}, j_{e}\right)$,

$$
\begin{gathered}
j_{s h}=-j_{s e}=\frac{\left(\rho_{h h}^{\mathrm{qp}}-\rho_{e h}^{\mathrm{qp}}\right) j_{h}-\left(\rho_{e e}^{\mathrm{qp}}-\rho_{e h}^{\mathrm{qp}}\right) j_{e}}{\rho_{e e}^{\mathrm{qp}}+\rho_{h h}^{\mathrm{qp}}-2 \rho_{e h}^{\mathrm{qp}}}, \\
\mathbf{j}_{n}=\mathbf{j}-\mathbf{j}_{s} \text {, and } \\
E_{h}=E_{e}=\rho_{c d}\left(j_{e}+j_{h}\right) .
\end{gathered}
$$


In Eq. (6) $\rho_{c d}$, which we will refer to as the condensate drag resistivity, is related to the components of the quasiparticle transresistivity and transconductivity matrices by

$$
\begin{aligned}
\rho_{c d} & =\frac{\rho_{e e}^{\mathrm{qp}} \rho_{h h}^{\mathrm{qp}}-\left(\rho_{e h}^{\mathrm{qp}}\right)^{2}}{\rho_{e e}^{\mathrm{qp}}+\rho_{h h}^{\mathrm{qp}}-2 \rho_{e h}^{\mathrm{qp}}} \\
& =\frac{1}{\sigma_{e e}^{\mathrm{qp}}+\sigma_{h h}^{\mathrm{qp}}+2 \sigma_{e h}^{\mathrm{qp}}} .
\end{aligned}
$$

Our conclusions thus far do not depend on the nature of the microscopic mechanism of superfluidity. We now turn our attention to the evaluation of $\rho_{c d}$ in a specific microscopic model. We will restrict our attention to the case where the particle densities in electron and hole layers are identical $\left(n_{e}=n_{h}=n\right)$ and use the BCS mean-field theory [12] to describe the pairing. This approach is qualitatively correct in the regime of high electron and hole densities, which is most accessible to experiments [13]. We will be able to express our results in terms of the solution to the mean-field gap equations at $P=0$. Taking the effective attractive interaction $V$ [14] which enters the BCS equations to be independent of momentum, the gap equations differ from their textbook counterparts $[12,15]$ only in that the the electron and hole masses $\left(m_{e}\right.$ and $\left.m_{h}\right)$ are not equal. The quasiparticle energies are given by $(\hbar=1)$

$$
\begin{aligned}
& E_{0 k}=E_{k}+\eta_{k}, \\
& E_{1 k}=E_{k}-\eta_{k},
\end{aligned}
$$

where $E_{k}=\left(\epsilon_{k}^{2}+\Delta^{2}\right)^{1 / 2}, \epsilon_{k}=\left(k^{2}-k_{F}^{2}\right) / 2 m_{+}, \eta_{k}=$ $\left(k^{2}-k_{F}^{2}\right) / 2 m_{-}, \quad 2 m_{ \pm}^{-1}=m_{e}^{-1} \pm m_{h}^{-1}, \quad$ and $\quad k_{F}=$ $(2 \pi n)^{1 / 2}$ is the Fermi momentum. The gap $\Delta$ is determined by solving the gap equation:

$$
\frac{1}{\lambda}=\int_{0}^{\omega_{c}} d \epsilon_{k} \frac{1}{\sqrt{\Delta^{2}+\epsilon_{k}^{2}}}\left[1-f\left(E_{0 k}\right)-f\left(E_{1 k}\right)\right] .
$$

In Eq. (9) $\lambda=N(0) V[N(0)$ is the density of states of free fermions of mass $m_{+}$and density $\left.n\right]$ is the usual dimensionless coupling constant $[12,15]$ of BCS theory, $f(E)=\left[\exp \left(E / k_{B} T\right)+1\right]^{-1}$, and $\omega_{c}$ is the cutoff for the attractive interaction.

The microscopic calculations, which are somewhat lengthy, are similar to common applications of BCS theory for superconductivity in metals $[12,15]$. The main steps of this calculation are sketched and the principal results are given below. We first calculate $n_{\text {pair }} / m_{\text {pair }}$ by evaluating the electron and hole currents to first order in the pairing momentum $P$ when the quasiparticles are in equilibrium with the condensate. For $m_{e}=m_{h}$ this calculation is identical to the calculation of the superfluid density which determines the penetration depth of a superconductor. We find that

$$
\frac{n_{\mathrm{pair}}}{m_{\mathrm{pair}}}=\frac{n_{e}-n_{n e}}{m_{e}}=\frac{n_{h}-n_{n h}}{m_{h}} .
$$

The equivalence of the two forms for the right hand side of Eq. (10) provides microscopic confirmation of the expectation, used in our minimum dissipation analysis, that the current carried by the electron-hole condensate is equal and opposite in electron and hole layers. In Eq. (10) the electron "normal" density $n_{n e}$ is given by

$$
\begin{aligned}
n_{n e}= & -\frac{1}{A} \sum_{k} \frac{k^{2}}{2 m_{+}}\left[u_{k}^{2} f^{\prime}\left(E_{0 k}\right)+v_{k}^{2} f^{\prime}\left(E_{1 k}\right)\right] \\
& -\frac{1}{A} \sum_{k} \frac{k^{2}}{2 m_{-}} \frac{\epsilon_{k}}{E_{k}}\left[u_{k}^{2} f^{\prime}\left(E_{0 k}\right)-v_{k}^{2} f^{\prime}\left(E_{1 k}\right)\right] \\
& -\frac{1}{A} \sum_{k} \frac{k^{2}}{4 m_{-}} \frac{\Delta^{2}}{E_{k}^{3}}\left[f\left(E_{0 k}\right)+f\left(E_{1 k}\right)-1\right],
\end{aligned}
$$

where $u_{k}^{2}=\left(1+\epsilon_{k} / E_{k}\right) / 2, v_{k}^{2}=\left(1-\epsilon_{k} / E_{k}\right) / 2$, and $A$ is the area of the layer. The normal hole density $n_{n h}$ is obtained by interchanging the indices $e$ and $h$, i.e., $E_{0 k} \leftrightarrow E_{1 k}$ and $m_{-} \rightarrow-m_{-}$in the above formula.

We have evaluated the quasiparticle transconductivity in the paired state in a single-loop approximation using the Nambu-Gorkov Green's function formalism. After some standard manipulations [16] this approximation leads to

$$
\sigma_{i j}^{\mathrm{qp}}=\frac{1}{A} \sum_{\vec{p}} \frac{\pi p^{2}}{2 m_{i} m_{j}} \int_{-\infty}^{\infty} f^{\prime}(\omega) A_{i j}^{2}(\vec{p}, \omega) d \omega,
$$

where $i$ and $j$ are layer indices,

$$
\mathbf{A}(\vec{p}, \omega) \equiv-\frac{1}{\pi} \operatorname{Im} \mathbf{G}(\vec{p}, \omega+i \delta),
$$

and $\mathbf{G}$ is the Nambu-Gorkov matrix Green's function. In the absence of disorder we have

$\mathbf{G}_{(0)}(\vec{p}, \omega+i \delta)=\frac{\left(\omega-\eta_{p}\right) \hat{1}+\epsilon_{p} \hat{\tau}_{3}+\Delta \hat{\tau}_{1}}{\left(\omega-E_{0 p}+i \delta\right)\left(\omega+E_{1 p}+i \delta\right)}$,

where the $\tau$ 's are Pauli matrices. To model disorder we have included a Born approximation [16] selfenergy correction $\boldsymbol{\Sigma}(\vec{p}, \omega)$ to the Nambu-Gorkov Green's function: $\mathbf{G}^{-1}(\vec{p}, \omega)=\mathbf{G}_{(0)}^{-1}(\vec{p}, \omega)-\mathbf{\Sigma}(\vec{p}, \omega)$. Assuming zero correlation length for the disorder potential in each layer and no correlation between the disorder in electron and hole layers a lengthy series of algebraic manipulations allows the quasiparticle transconductivity to be expressed in terms of the normal state scattering times for electron and hole layers $\tau_{n e}$ and $\tau_{n h}$. We find that

$$
\frac{\sigma_{h h}^{\mathrm{qp}}}{\sigma_{0}}=\frac{(1+y)^{2}}{2 \alpha} \int_{0}^{\infty} d \epsilon_{k}\left[\frac{u_{k}^{4} \tilde{\tau}_{0 k}}{\cosh ^{2}\left(E_{0 k} / 2 k_{B} T\right)}+\frac{v_{k}^{4} \tilde{\tau}_{1 k}}{\cosh ^{2}\left(E_{1 k} / 2 k_{B} T\right)}\right],
$$




$$
\begin{aligned}
\frac{\sigma_{e e}^{\mathrm{qp}}}{\sigma_{0}} & =\frac{(1-y)^{2}}{2 \alpha} \int_{0}^{\infty} d \epsilon_{k}\left[\frac{v_{k}^{4} \tilde{\tau}_{0 k}}{\cosh ^{2}\left(E_{k 0} / 2 k_{B} T\right)}+\frac{u_{k}^{4} \tilde{\tau}_{1 k}}{\cosh ^{2}\left(E_{1 k} / 2 k_{B} T\right)}\right], \\
\frac{\sigma_{e h}^{\mathrm{qp}}}{\sigma_{0}} & =-\frac{1-y^{2}}{2 \alpha} \int_{0}^{\infty} d \epsilon_{k} u_{k}^{2} v_{k}^{2}\left[\frac{\tilde{\tau}_{0 k}}{\cosh ^{2}\left(E_{0 k} / 2 k_{B} T\right)}+\frac{\tilde{\tau}_{1 k}}{\cosh ^{2}\left(E_{1 k} / 2 k_{B} T\right)}\right],
\end{aligned}
$$

where $y \equiv\left(m_{e}-m_{h}\right) /\left(m_{h}+m_{e}\right), \quad r \equiv\left(\tau_{n e}-\tau_{n h}\right) /$ $\left(\tau_{n h}+\tau_{n e}\right), \alpha=(1+y) /(1+r)+(1-y) /(1-r)$, $2\left(\tau_{n}\right)^{-1} \equiv \tau_{n h}^{-1}+\tau_{n e}^{-1}, \sigma_{0} \equiv n e^{2} \tau_{n} \alpha / m_{+}$, and $\tilde{\tau}_{0 k}$ and $\tilde{\tau}_{1 k}$ are the energy dependent scattering times which give the lifetimes of the quasiparticle states in units of $\tau_{n}$ :

$$
\begin{aligned}
& \tilde{\tau}_{0 k}=\frac{\left|\epsilon_{k} / E_{k}+y\right|}{u_{k}^{4}(1+y)(1+r)+v_{k}^{4}(1-y)(1-r)}, \\
& \tilde{\tau}_{1 k}=\frac{\left|\epsilon_{k} / E_{k}-y\right|}{u_{k}^{4}(1-y)(1-r)+v_{k}^{4}(1+y)(1+r)} .
\end{aligned}
$$

In Fig. 1 we show numerical results calculated from the above expressions for the values $m_{h} / m_{e}=7.57$, $\tau_{n e} / \tau_{n h}=m_{h} / m_{e}$ appropriate to electrons and holes in GaAs, with equal concentrations of impurities in the two layers. (The precise values of the parameters have no qualitative importance.) The drag resistivity $\rho_{c d} \equiv$ $\sigma_{c d}^{-1}$ immediately jumps to a value comparable to the normal state resistivity $\left(\sim \sigma_{0}^{-1}\right)$ at $T_{c}$ and it diverges exponentially as the temperature goes to zero. In this limit the quasiparticle conductivity vanishes because of the small number of thermally excited quasiparticles. Consequently, a larger and larger electric field is required to drive the normal component of the current. We also plot the behavior of the quasiparticle conductivities $\sigma_{e e}^{\mathrm{qp}}$, $\sigma_{h h}^{\mathrm{qp}}$, and $\sigma_{e h}^{\mathrm{qp}}$ in the superfluid phase. The quasiparticle transconductivity $\sigma_{e h}^{\mathrm{qp}}$ vanishes as $T \rightarrow T_{c}$ because, in our theory, we include no correlation between the two

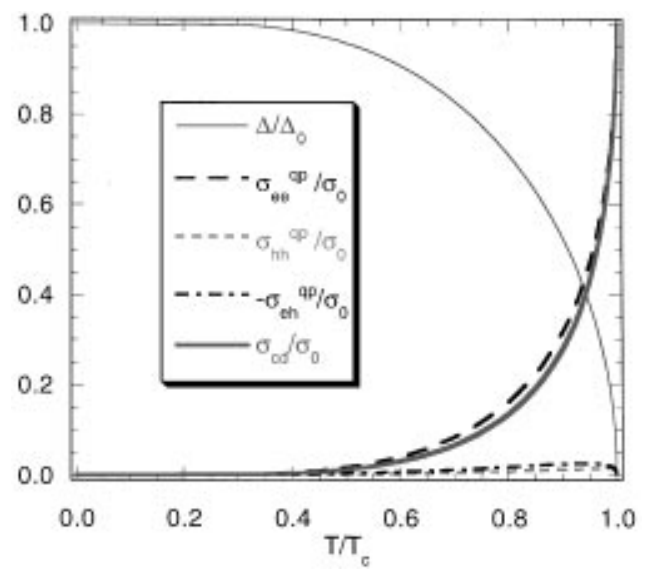

FIG. 1. Ratio of the BCS model condensate drag conductivity $\sigma_{c d}=\sigma_{e e}^{\mathrm{qp}}+\sigma_{h h}^{\mathrm{qp}}+2 \sigma_{e h}^{\mathrm{qp}}=\rho_{c d}^{-1}$ to the normal doublelayer conductivity $\sigma_{0}$ as a function of $T / T_{c}$ for GaAs $\left(m_{h} / m_{e}=7.57\right.$ and $\left.\tau_{n e} / \tau_{n h}=m_{h} / m_{e}\right)$. Also plotted quasiparticle conductivities $\sigma_{e e}^{\mathrm{qp}} / \sigma_{0}, \sigma_{h h}^{\mathrm{qp}} / \sigma_{0}$, and $-\sigma_{e h}^{\mathrm{qp}} / \sigma_{0}$, and BCS gap $\Delta$ in units of its zero temperature value $\Delta_{0}$. Qualitatively similar results are obtained for different values of the parameters. layers other than the one implied by the existence of the electron-hole condensate.

Our microscopic calculations are based on the BCS scenario of the superfluid transition. This is the most likely scenario for electron-hole double layers at densities of experimental interest. At lower densities other routes are possible. For example, one could have exciton pair formation in the normal state, followed by Bose-Einstein condensation at lower temperature. In this scenario, the transresistivity would be large even in the normal state, due to the strong electron-hole correlation. In the superfluid state Eq. (6) would still hold, following merely from the possibility of dissipation-free conduction. Arguments similar to those presented earlier lead us to conclude that even in this case the transresistivity would increase discontinuously at the condensation temperature, although the size of the jump could be considerably smaller than in the high density case.

In the two-dimensional electron-hole layers, considered in this Letter, the superfluid transition is expected to be of the Kosterlitz-Thouless type. This means that our microscopic theory may require quantitative correction in a region near the transition temperature where fluctuations are important. In particular, the "jump" in the transresistivity could be distributed over a finite temperature range. At lower temperatures our macroscopic analysis shows that the qualitative behavior of the transresistivity is completely independent of microscopic details. We conclude that transresistance measurements should provide a foolproof test for the presence of a superfluid condensate.

In closing we note that the analysis presented here is based on the linear approximation for the response of quasiparticles to an electric field. This approximation is justified for sufficiently weak electric fields, i.e., fields satisfying the condition $e E \xi \ll \Delta$, where $\xi$ is the coherence length. In terms of the current flowing in one layer (with the other layer kept in an open circuit) this condition takes the form [see Eq. (6), with $j_{h}=0$ ] $j_{e} / j_{c} \ll \min \left\{1, \frac{n_{n}}{n} \frac{v_{F} \tau}{\xi}\right\}$, where $j_{c}=n \hbar / m \xi$ is the thermodynamic critical current, $\tau$ is the elastic scattering time of quasiparticles, $v_{F}$ is the Fermi velocity, and $n_{n}$ is the "normal fluid" density. Thus, at any finite temperature below $T_{c}$, there is a finite range of weak currents in which our linear response approximation is valid. However, the restriction becomes more and more stringent as $T \rightarrow T_{c}$ and $\xi \rightarrow \infty$. A theoretical investigation of nonlinear effects remains an interesting problem for the future.

This work was supported by NSF Grants No. DMR9416906 and No. DMR-9403908. G. V. acknowledges the kind hospitality of the Condensed Matter Theory 
Group at Indiana University, where this work was initiated. We also acknowledge useful discussions with Leo Radzihovsky and L. Swierkowski.

[1] S. I. Shevchenko, Fiz. Nizk. Temp. 2, 505 (1976) [Sov. J. Low Temp. Phys. 2, 251 (1976)]; Yu. E. Lozovik and V. I. Yudson, Pis'ma Zh. Eksp. Teor. Fiz. 22, 556 (1975) [JETP Lett. 22, 271 (1975)]; Zh. Eksp. Teor. Fiz. 71, 738 (1976) [Sov. Phys. JETP 44, 389 (1976)].

[2] T. Fukuzawa, E. E. Mendez, and J. M. Hong, Phys. Rev. Lett. 64, 3066 (1990).

[3] J.A. Kash, M. Zachau, E. Mendez, J. M. Hong, and T. Fukuzawa, Phys. Rev. Lett. 66, 2247 (1991).

[4] U. Sivan, P. M. Solomon, and H. Shtrikman, Phys. Rev. Lett. 68, 1196 (1992).

[5] B. E. Kane, J.P. Eisenstein, W. Wegscheider, L. N. Pfeiffer, and K. W. West, Appl. Phys. Lett. 65, 3266 (1994).

[6] Y. Kuramoto and C. Horie, Solid State Commun. 25, 713 (1979); I. V. Lerner and Yu. E. Lozovik, Zh. Eksp. Teor. Fiz. 80, 1488 (1981) [Sov. Phys. JETP 53, 763 (1981)]; Y. A. Bychkov and E. I. Rashba, Solid State Commun. 48, 399 (1983); D. Paquet, T. M. Rice, and K. Ueda, Phys. Rev. B 32, 5208 (1985).

[7] At strong fields there is an exact mapping between electron-electron and electron-hole double-layer systems: A.H. MacDonald and E.H. Rezayi, Phys. Rev. B 42, 3224 (1990); A. B. Dzyubenko and Yu. E. Lozovik, Fiz. Tverd. Tela (Leningrad) 25, 1519 (1983) [Sov. Phys. Solid State 25, 874 (1983)]. The excitonic condensate state of the electron-hole system maps to a state with $X Y$ pseudospin ferromagnetism in the electron-electron system. The Kosterlitz-Thouless transition temperature for this phase has been estimated to be as large as $\sim 0.5 \mathrm{~K}$ : K. Moon et al., Phys. Rev. B 51, 5138 (1995). For a review of broken symmetries of electron-electron doublelayer systems in strong magnetic fields, see S. M. Girvin and A. H. MacDonald, in Novel Quantum Liquids in LowDimensional Semiconductor Structures, edited by S. Das Sarma and Aron Pinczuk (Wiley, New York, 1995).

[8] M. B. Pogrebinskii, Fiz. Tekh. Poluprovodn. 11, 637 (1977) [Sov. Phys. Semicond. 11, 372 (1977)]; Peter J. Price, Physica (Amsterdam) 117B \& 118B, 750 (1983).
[9] H.C. Tso, P. Vasilopolous, and F. M. Peeters, Phys. Rev. Lett. 68, 2516 (1992); 70, 2146 (1993); A.-P. Jauho and H. Smith, Phys. Rev. B 47, 4420 (1993); Lian Zheng and A.H. MacDonald, Phys. Rev. B 48, 8203 (1993); Karsten Flensberg and Ben Yu-Kuang Hu, Phys. Rev. Lett. 73, 3572 (1994); Karsten Flensberg, B. Y.-K Hu, A.-P. Jahuo, and J. M. Kinaret, Report No. cond-mat 950409-2, 1995 (to be published); Alex Kamenev and Yuval Oreg, Phys. Rev. B 52, 7516 (1995); Martin Bonsager and A.-P. Jauho (to be published); L. Swierkowski, J. Szymanski, and Z.W. Gortel, Phys. Rev. Lett. 74, 3245 (1995); E. Shimshoni and S. L. Sondhi, Phys. Rev. B 49, 11484 (1994).

[10] T. J. Gramila, J.P. Eisenstein, A. H. MacDonald, L. N. Pfeiffer, and K.W. West, Phys. Rev. Lett. 66, 1216 (1991); T. J. Gramila, J. P. Eisenstein, A. H. MacDonald, L. N. Pfeiffer, and K. W. West, Surf. Sci. 263, 446 (1992); T. J. Gramila, J.P. Eisenstein, A.H. MacDonald, L. N. Pfeiffer, and K. W. West, Phys. Rev. B 47, 12957 (1993); see also P. M. Solomon, P. J. Price, D. J. Frank, and D. C. La Tulipe, Phys. Rev. Lett. 63, 2508 (1989).

[11] S. R. DeGroot and P. Mazur, Non-Equilibrium Thermodynamics (Dover Publications, Inc., New York, 1962), Chap. 5; I. Prigogine, Introduction to Thermodynamics of Irreversible Processes (Wiley, New York, 1961).

[12] See, for example, Michael Tinkham, Introduction to Superconductivity (McGraw-Hill, New York, 1975); P. G. de Gennes, Superconductivity of Metals and Alloys (Benjamin, New York, 1966).

[13] At lower electron-hole density we enter a regime in which strong correlation effects (leading, for instance, to the formation of excitons in the normal state) and disorderinduced localization must be taken into account.

[14] In our calculation we assume that BCS theory is approximately valid for electron-hole systems but treat the effective interation which enters the theory, which is difficult to determine reliably as a phenomenological parameter.

[15] J. R. Schrieffer, Theory of Superconductivity (Benjamin, New York, 1983); see also Superconductivity, edited by R. W. Parks (Dekker, New York, 1969).

[16] G.D. Mahan, Many-Particle Physics (Plenum Press, New York, 1990), Chap. 9. 